\title{
Customised Cranioplasty Implant for Decompressive Craniectomy Patients - A Technical Note
}

\author{
Kaif MOHAMMAD \\ Doctor Ram Manohar Lohia Institute of Medical Sciences, Department of Neurosurgery, Lucknow, India
}

\section{ABSTRACT}

Cranioplasty is a commonly performed procedure following decompressive craniectomy. The patients are usually young earning persons and increasing cost of treatment is difficult to bear especially in low and middle income countries. A variety of options are available for cranioplasty ranging from autologus bone graft to xenograft and synthetic materials. Presently the autologous cryopreserved or subcutaneously preserved bone graft is considered as the preferred choice, but it has the drawbacks of bone resorption and infection. Custom made titanium implants and polyetheretherketone implants, which are manufactured using 3D printing technologies, are also being used. However, their use is limited because of high cost and lack of availability of these technologies in the developing world, which have the maximum number of such cases. The authors present an easy and low cost technique for making a customised cranioplasty implant by polymethylmethacrylate using the craniotomy bone flap of the patient as a template.

KEYWORDS: Cranioplasty, Decompressive craniectomy, Polymethylmethacrylate

\section{INTRODUCTION}

$\mathrm{D}$ ecompressive craniectomy is a commonly performed procedure in cases of raised intracranial pressure following road traffic accidents (RTA) and is followed by cranioplasty at a later date. The most common indication for cranioplasty is decompressive craniectomy in head injury patients. The victims are mostly the young earning members of the family. Low and middle income countries like India constitute $90 \%$ of the burden of traumatic brain injury (TBI). Without insurance or state sponsored specialised care, the cost of the treatment is borne by the patient's family (5).

There are a number of options available for cranioplasty from autograft to xenografts and synthetic materials. The ideal material for cranioplasty as described in the literature should be radiolucent, resistant to infection, not conductive of heat or cold, resistant to biomechanical processes, malleable, inexpensive and ready to use.
There is historical evidence of cranioplasty for which different materials were used ranging from bone to xenografts to gold and silver plates (4). Presently the autologous cryopreserved or subcutaneously preserved bone graft is considered as the preferred choice (2). However, it is associated with complications. It has been reported that cryopreserved bone graft has higher rate of resorption and infection. The other disadvantages are that till date the method of cryopreservation has not been standardized and it also incurs a high cost. The subcutaneously stored bone graft is a low cost option but it has the disadvantages of morbidity of a separate incision, physical discomfort to the patient and resorption of bone graft.

Various synthetic materials have been used for cranioplasty. Polymethylmethacrylate (PMMA) is a commonly used material as a bone graft. The advantages of PMMA are that the long-term favourable results are available, but it has the disadvantages regarding proper moulding during surgery and exothermic burn reaction. 
To overcome the disadvantages of intraoperative accurate moulding, custom made titanium implants (1) and polyetheretherketone (PEEK) implants, which are manufactured using 3D printing technologies (3), are being used. However, their use is limited because of high cost and lack of availability in the developing world, which have the maximum number of such cases.

\section{TECHNIQUE FOR MAKING OF CUSTOMISED CRANIOPLASTY IMPLANT}

In order to overcome the shortcomings of high cost and difficulty of intraoperative moulding, the authors used the bone flap of the patient harvested during the decompressive craniectomy as a template. This bone flap was used as a template to construct a C-silicone mould. This C-silicone mould was used to construct the bone flap for cranioplasty

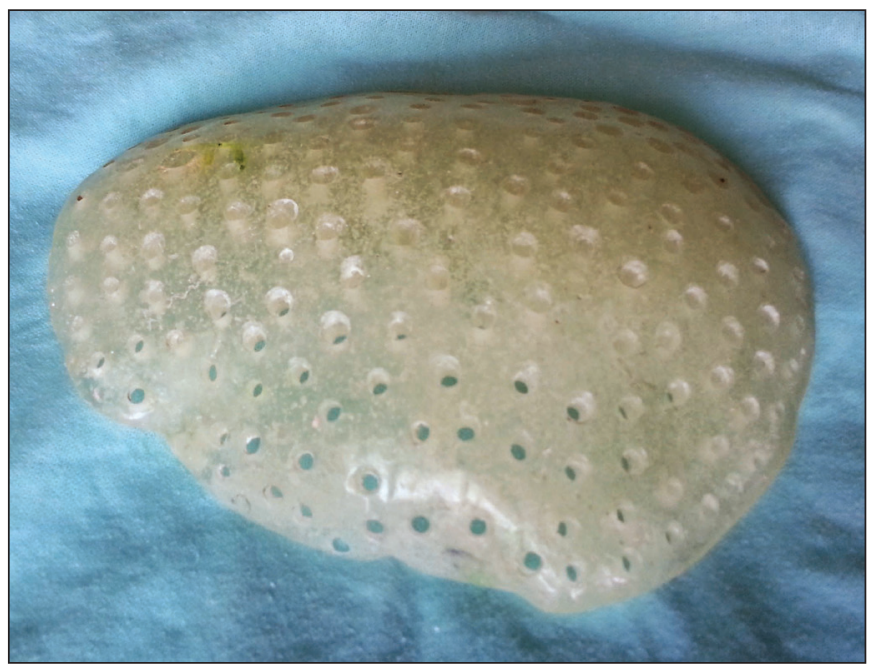

Figure 1: Customised PMMA cranioplasty implant.

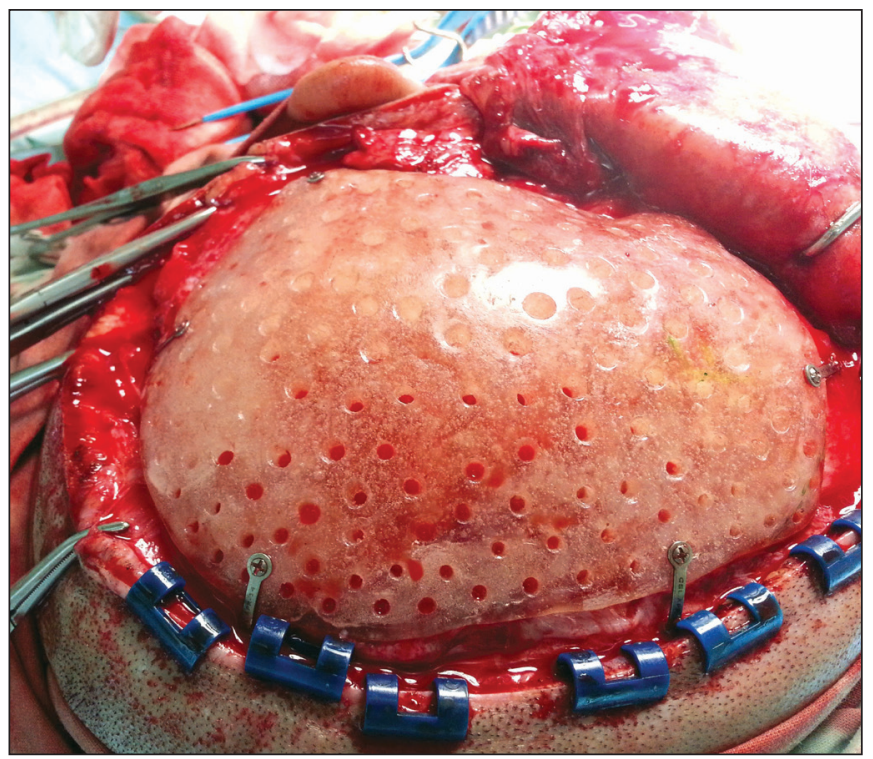

Figure 2: Cranioplasty implant fixed in situ. by slowly packing it with PMMA. This resulted in the creation of a PMMA duplicate to be used as a cranioplasty implant. Approximately 40 minutes after packing the C-silicone mould with PMMA, the PMMA cranioplasty implant was removed from the C-Silicone. Now the sharp margins of the PMMA duplicate were trimmed and multiple small burr holes were created to avoid any collection beneath the implant postoperatively. The ethylene oxide sterilization method was used to sterilize the implant (Figure 1).

\section{TECHNIQUE FOR IMPLANTING THE CUSTOMISED CRANIOPLASTY IMPLANT}

Preoperative examination was done to exclude hydrocephalus by looking for any bulge at the operation site and a fresh computed tomography (CT) scan was done. The patient was taken up under general anaesthesia and positioned in desired position. The skin was infiltrated with diluted adrenaline solution (1:800000) to avoid excessive bleeding. The skin was carefully dissected from the underlying tissue to avoid arachnoid breach and cerebrospinal fluid (CSF) leak. If any breach occurred, it was repaired using 4-0 poly-propylene suture. The bone defect was defined and its entire margin cleared of the adhesions. Now the customised PMMA implant was taken and fixed to the defect using titanium screws and plate (Figure 2). Haemostasis was secured and the wound was thoroughly washed with normal saline. The skin was closed in the standard manner, and a subgaleal drain, which was removed after 24 hours, was inserted.

\section{ADVANTAGES}

The advantages of this procedure are that we get an exact replica of the craniectomy bone defect so it has a better cosmetic result. Secondly the injury due to exothermic reaction during intraoperative moulding of bone flap is avoided in this procedure. The most important advantage is of cost reduction. Similar customised implants of PEEK and titanium are available, but their costs are very high in comparison to this implant which costs approximately 75 USD.

\section{CONCLUSION}

Customised cranioplasty implants are efficient means for cranioplasty. 3D printing and customised titanium implants for cranioplasty are being used, but their use is limited due to its high cost and lack of availability in the developing countries. PMMA, which is a widely used material for cranioplasty, has proven long-term safety results. This method uses the native craniotomy bone as a mould, has the advantage of low cost, established safety of PMMA, ease of availability of material, no dependence on high end technology and good cosmetic results.

\section{- REFERENCES}

1. Höhne J, Brawanski A, Gassner HG, Schebesch KM: Feasibility of the custom-made titanium cranioplasty CRANIOTOP®. Surg Neurol Int 4: 88, 2013 
2. Kim BJ, Hong KS, Park KJ, Park DH, Chung YG, Kang SH: Customized cranioplasty implants using three-dimensional printers and polymethyl-methacrylate casting. J Korean Neurosurg Soc 52: 541-546, 2012

3. Ng ZY, Nawaz I: Computer-designed PEEK implants: A peek into the future of cranioplasty. J Craniofac Surg 25(1): e55-e58, 2014
4. Shah $A M$, Jung $H$, Skirboll S: Materials used in cranioplasty: $A$ history and analysis Neurosurg Focus 36 (4): E19, 2014

5. Sinha S, Raheja A, Garg M, Moorthy S, Agrawal D, Gupta DK, Satyarthee GD, Singh PK, Borkar SA, Gurjar H, Tandon V, Pandey RM, Sharma BS: Decompressive craniectomy in traumatic brain injury: A single center, multivariate analysis of 1,236 patients at a tertiary care hospital in India. Neurol India 63(2): 175-182, 2015 